\title{
On an hypercomplex generalization of Gould-Hopper and related Chebyshev polynomials
}

\author{
I. Cação and H.R. Malonek \\ Departamento de Matemática, Universidade de Aveiro, \\ isabel.cacao@ua.pt, hrmalon@ua.pt
}

\begin{abstract}
An operational approach introduced by Gould and Hopper to the construction of generalized Hermite polynomials is followed in the hypercomplex context to build multidimensional generalized Hermite polynomials by the consideration of an appropriate basic set of monogenic polynomials. Directly related functions, like Chebyshev polynomials of first and second kind are constructed.
\end{abstract}

Keywords: hypercomplex function theory, exponential operators, generalized Hermite polynomials, Chebyshev polynomials

\section{Introduction}

Gould and Hopper [10] defined the generalized Hermite polynomials $H_{k, m}^{\lambda}$ of order $m$ and parameter $\lambda$ by the operational identity

$$
H_{k, m}^{\lambda}(x):=e^{\lambda\left(\frac{d}{d x}\right)^{m}} x^{k}, \quad x \in \mathbb{R} .
$$

Multidimensional analogues can be defined in the hypercomplex context of generalized holomorphic function theory by considering an appropriate hypercomplex exponential operator and a basic set of polynomials that can replace $x^{k}$. Generalized holomorphic function theory (more frequently called monogenic function theory) generalizes to higher dimensions the theory of holomorphic functions of one complex variable by using Clifford algebras. One significant difference to the complex case is that in higher dimensions the set of monogenic functions is not closed with respect to the usual multiplication. This aspect lead us to the essential question: how to replace $x^{k}$ ? As the sequence $\left(x^{k}\right)_{k \in \mathbb{N}}$ is an Appell sequence with respect to the derivative operator $D:=\frac{d}{d x}$ involved in (1), one can consider for this replacement a monogenic Appell sequence with respect to the hypercomplex derivative operator (for the hypercomplex derivative of a monogenic function, see [11], based on the previous work about hypercomplex differentiability contained in [13]). In this work we consider the monogenic Appell sequence defined in $[9,15]$ that contains the usual real and complex powers as particular cases. 
The multidimensional counterpart in hypercomplex function theory of the usual exponential operator is defined through the monogenic exponential function considered in $[9,15]$ that is based naturally on the constructed Appell sequence. Those analytic tools allow us to follow the operational approach (1) to the construction of Gould-Hopper polynomials in the context of hypercomplex function theory. A similar operational approach was already considered in [5] to obtain monogenic generalized Laguerre polynomials.

The paper is organized as follows: in Section 2 the necessary basic notions of Clifford analysis are introduced briefly and in sections 3 and 4 we prepare the operational approach to generalize Hermite polynomials based on the hypercomplex counterpart of (1), which is the subject of Section 5. Finally in Section 6, we establish a natural link between the constructed Gould-Hopper polynomials and the monogenic Chebyshev polynomials of first and second kinds.

\section{Basic notions}

Let $\left\{e_{1}, e_{2}, \cdots, e_{n}\right\}$ be an orthonormal basis of the Euclidean vector space $\mathbb{R}^{n}$ with the non-commutative multiplication rule

$$
e_{k} e_{l}+e_{l} e_{k}=-2 \delta_{k l}, k, l=1, \cdots, n,
$$

where $\delta_{k l}$ is the Kronecker symbol. The set $\left\{e_{A}: A \subseteq\{1, \cdots, n\}\right\}$ with

$$
e_{A}=e_{h_{1}} e_{h_{2}} \cdots e_{h_{r}}, 1 \leq h_{1} \leq \cdots \leq h_{r} \leq n, e_{\emptyset}=e_{0}=1
$$

forms a basis of the $2^{n}$-dimensional Clifford algebra $\mathcal{C} \ell_{0, n}$ over $\mathbb{R}$. Let $\mathbb{R}^{n+1}$ be embedded in $\mathcal{C} \ell_{0, n}$ by identifying $\left(x_{0}, x_{1}, \cdots, x_{n}\right) \in \mathbb{R}^{n+1}$ with the algebra's element $x=x_{0}+\underline{x} \in \mathcal{A}:=\operatorname{span}_{\mathbb{R}}\left\{1, e_{1}, \ldots, e_{n}\right\} \subset \mathcal{C} \ell_{0, n}$. The elements of $\mathcal{A}$ are called paravectors and $x_{0}=\operatorname{Sc}(x)$ and $\underline{x}=\operatorname{Vec}(x)=e_{1} x_{1}+\cdots+e_{n} x_{n}$ are the so-called scalar resp. vector part of the paravector $x$. The conjugate of $x$ is given by $\bar{x}=x_{0}-\underline{x}$ and the norm $|x|$ of $x$ is defined by $|x|^{2}=x \bar{x}=$ $\bar{x} x=x_{0}^{2}+x_{1}^{2}+\cdots+x_{n}^{2}$. We consider functions of the form $f(z)=\sum_{A} f_{A}(z) e_{A}$, where $f_{A}(z)$ are real valued, i.e. $\mathcal{C} \ell_{0, n}$-valued functions defined in some open subset $\Omega \subset \mathbb{R}^{n+1}$. Continuity and real differentiability of $f$ in $\Omega$ are defined componentwise. The generalized Cauchy-Riemann operator in $\mathbb{R}^{n+1}, n \geq 1$, is defined by

$$
\bar{\partial}:=\partial_{0}+\partial_{\underline{x}}
$$

where

$$
\partial_{0}:=\frac{\partial}{\partial x_{0}}, \quad \partial_{\underline{x}}:=e_{1} \frac{\partial}{\partial x_{1}}+\cdots+e_{n} \frac{\partial}{\partial x_{n}} .
$$

The higher dimensional analogue to an holomorphic function is now a $C^{1}(\Omega)$ function $f$ satisfying the equation

$$
\bar{\partial} f=0 \quad(\operatorname{resp} \cdot f \bar{\partial}=0)
$$

and it is called left monogenic (resp. right monogenic). 
We suppose that $f$ is hypercomplex differentiable in $\Omega$ in the sense of [11, 13], i.e. it has a uniquely defined areolar derivative $f^{\prime}$ in each point of $\Omega$ (see also [14]). Then $f$ is real differentiable and $f^{\prime}$ can be expressed by

$$
f^{\prime}=\frac{1}{2} \partial
$$

where $\partial:=\partial_{0}-\partial_{\underline{x}}$ is the conjugate Cauchy-Riemann operator. Since a hypercomplex differentiable function belongs to the kernel of $\bar{\partial}$, it follows that in fact $f^{\prime}=\partial_{0} f$ like in the complex case.

\section{Basic homogeneous monogenic polynomial sequence}

In this section, we consider a special set of monogenic basis functions defined and studied in $[8,9,15]$, namely functions of the form

$$
\mathcal{P}_{k}^{n}(x)=\sum_{s=0}^{k} T_{s}^{k}(n) x^{k-s} \bar{x}^{s},
$$

where

$$
T_{s}^{k}(n)=\left(\begin{array}{l}
k \\
s
\end{array}\right) \frac{\left(\frac{n+1}{2}\right)_{(k-s)}\left(\frac{n-1}{2}\right)_{(s)}}{(n)_{k}},
$$

and $a_{(r)}$ denotes the Pochhammer symbol, i.e. $a_{(r)}=\frac{\Gamma(a+r)}{\Gamma(a)}$, for any integer $r>1$, and $a_{(0)}:=1$.

We remark that $\mathcal{P}_{0}^{n}(x)=1$ and $\mathcal{P}_{k}^{n}(0)=0, k>0$, in consequence of the homogeneity of these functions. Moreover, for each $k \geq 1, \mathcal{P}_{k}^{n}$ is a polynomial of degree of homogeneity exactly $k$ and under the additional (but natural) condition $\mathcal{P}_{k}{ }^{n}(1)=1$, it holds (see [9])

$$
\frac{1}{2} \partial \mathcal{P}_{k}^{n}=k \mathcal{P}_{k-1}^{n}, \quad k \geq 1 .
$$

This means that $\left(\mathcal{P}_{k}{ }^{n}\right)_{k \in \mathbb{N}}$ is an Appell sequence.

\section{Particular cases:}

1. Consider $\underline{x}=0$. Taking into account that $\sum_{s=0}^{k} T_{s}^{k}(n)=1$, we get $\mathcal{P}_{k}{ }^{n}(x)=x_{0}^{k}$, i.e., $\mathcal{P}_{k}{ }^{n}$ are the usual powers in the real variable $x_{0}$, for each $\mathrm{k}=0,1,2, \ldots$. Notice that this case can be formally included in the above definitions as the case $n=0$, with $T_{0}^{k}(0)=1$ and $T_{s}^{k}(0)=0$, for $0<s \leq k$.

2. Consider $x_{0}=0$. Then we obtain the essential property, which characterizes the difference to the complex case,

$$
\mathcal{P}_{k}^{n}(\underline{x})=c_{k}(n) \underline{x}^{k},
$$


where

$$
c_{k}(n):=\sum_{s=0}^{k}(-1)^{s} T_{s}^{k}(n)= \begin{cases}\frac{k ! !(n-2) ! !}{(n+k-1) ! !}, & \text { if } k \text { is odd } \\ c_{k-1}(n), & \text { if } k \text { is even }\end{cases}
$$

and $c_{0}(n)=1$. As usual, we define $(-1) ! !=0 ! !=1$.

Using equality (4), the binomial-type formula for this Appell sequence (see [5]) can be written as

$$
\begin{aligned}
\mathcal{P}_{k}^{n}(x) & =\sum_{s=0}^{k}\left(\begin{array}{l}
k \\
s
\end{array}\right) x_{0}^{k-s} \mathcal{P}_{s}^{n}(\underline{x}) \\
& =\sum_{s=0}^{k}\left(\begin{array}{l}
k \\
s
\end{array}\right) c_{s}(n) x_{0}^{k-s} \underline{x}^{s} .
\end{aligned}
$$

From the above representation, we can easily compute the first polynomials:

$$
\begin{aligned}
\mathcal{P}_{0}^{n}(x) & =1 & \mathcal{P}_{1}^{n}(x) & =x_{0}+\frac{1}{n} \underline{x} \\
\mathcal{P}_{2}^{n}(x) & =x_{0}^{2}+\frac{2}{n} x_{0} \underline{x}+\frac{1}{n} \underline{x}^{2} & \mathcal{P}_{3}^{n}(x) & =x_{0}^{3}+\frac{3}{n} x_{0}^{2} \underline{x}+\frac{3}{n} x_{0} \underline{x}^{2}+\frac{3}{n(n+2)} \underline{x}^{3} .
\end{aligned}
$$

We observe that in the complex case $(n=1)$, the polynomials $\mathcal{P}_{k}^{1}$ coincide, as expected, with the usual powers $z^{k}$. In fact, from $(5)$, we get $c_{k}(1)=1$, for all $k$. Then, the binomial-type formula (6) permits to state that

$$
\mathcal{P}_{k}^{1}(x)=\sum_{s=0}^{k}\left(\begin{array}{l}
k \\
s
\end{array}\right) x_{0}^{k-s} \underline{x}^{s}=\left(x_{0}+e_{1} x_{1}\right)^{k} \simeq z^{k} .
$$

We remark that the study of Appell sequences in the hypercomplex context started in $[9,15]$ and it has been object of interest in recent years $([1,6,12])$ for different purposes.

\section{Monogenic exponential function}

The existence of a generalized holomorphic exponential function was from the beginning on in Clifford analysis a principal question. The first attempts towards a meaningful definition of an exponential function in the context of Clifford analysis have been $[2,17]$ and both papers rely on the Cauchy-Kowalevskaya extension approach (see also [4]), starting from the exponential function with imaginary argument and asking for a monogenic function which restriction to the real axis 
equals to the exponential function with real argument. Another possibility, motivated by the fact that hypercomplex differentiability is granted for monogenic functions, is to use the hypercomplex derivative of a monogenic function. This approach was followed in $[6,9,15]$ to define a monogenic exponential function $f$ as a solution of the simple first order differential equation $f^{\prime}=f$, with $f(0)=1$, where $f^{\prime}$ stands for the hypercomplex derivative of $f$. The combination of this approach with the constructed Appell sequence $\left(\mathcal{P}_{k}{ }^{n}\right)_{k \in \mathbb{N}}$ leads to the monogenic exponential function in $\mathbb{R}^{n+1}$ defined by

$$
\operatorname{Exp}_{n}(x)=\sum_{k=0}^{\infty} \frac{\mathcal{P}_{k}{ }^{n}(x)}{k !}
$$

Considering $\omega(x):=\frac{\underline{x}}{|\underline{x}|}$ with $\omega^{2}=-1$ as the equivalent for the imaginary unit $i$, a closed formula for the monogenic exponential (7) in terms of Bessel functions of integer or half-integer orders (depending on the dimension $n$ ) was given in [9]:

Theorem 1. The $\operatorname{Exp}_{n}$-function can be written in terms of Bessel functions of the first kind, $J_{a}(x)$, for orders $a=\frac{n}{2}-1, \frac{n}{2}$ as

$$
\operatorname{Exp}_{n}\left(x_{0}+\underline{x}\right)=e^{x_{0}} \Gamma\left(\frac{n}{2}\right)\left(\frac{2}{|\underline{x}|}\right)^{\frac{n}{2}-1}\left(J_{\frac{n}{2}-1}(|\underline{x}|)+\omega(x) J_{\frac{n}{2}}(|\underline{x}|)\right) .
$$

Let $U_{1}$ and $U_{2}$ be (right) linear modules over $\mathcal{C} \ell_{0, n}$ and $\hat{T}: U_{1} \rightarrow U_{2}$ be a hypercomplex (right) linear operator. The exponential function in $\mathbb{R}^{n+1}$ defined above permits to consider the exponential (right) operator

$$
\operatorname{Exp}_{n}(\lambda \hat{T})=\sum_{k=0}^{\infty} \frac{\mathcal{P}_{k}{ }^{n}(\hat{T})}{k !} \lambda^{k}, \lambda \in \mathbb{R}
$$

as a multidimensional counterpart in hypercomplex function theory of the usual exponential operator $e^{\lambda Q}=\sum_{k=0}^{\infty} \frac{Q^{k}}{k !} \lambda^{k}$.

\section{Monogenic generalized Hermite polynomials}

We consider the exponential operator $\operatorname{Exp}_{n}\left(\lambda\left(\frac{1}{2} \partial\right)^{m}\right)$ applied to the Appell sequence $\left(\mathcal{P}_{k}{ }^{n}(x)\right)_{k \geq 0}$ as a counterpart of Gould-Hoppers' operational approach (1) to define the hypercomplex generalized Hermite polynomials $H_{k, m}^{(\lambda)}$ of integer order $m$ and real parameter $\lambda$ as

$$
\begin{aligned}
H_{k, m}^{(\lambda)}(x) & :=\operatorname{Exp}_{n}\left(\lambda\left(\frac{1}{2} \partial\right)^{m}\right)\left(\mathcal{P}_{k}{ }^{n}(x)\right) \\
& =\sum_{r=0}^{\infty} \frac{1}{r !} \mathcal{P}_{r}{ }^{n}\left(\lambda\left(\frac{1}{2} \partial\right)^{m}\right) \mathcal{P}_{k}{ }^{n}(x) \\
& =\sum_{r=0}^{\infty} \frac{1}{r !} \frac{\lambda^{r}}{2^{r m}} \mathcal{P}_{r}{ }^{n}\left(\partial^{m}\right) \mathcal{P}_{k}{ }^{n}(x) .
\end{aligned}
$$


Notice that

$$
\begin{aligned}
\partial^{m} & =\left(\partial_{0}-\partial_{\underline{x}}\right)^{m} \\
& =\sum_{j=0}^{m}\left(\begin{array}{c}
m \\
j
\end{array}\right) \partial_{0}^{m-j}\left(-\partial_{\underline{x}}\right)^{j} \\
& =\sum_{i=0}^{\left[\frac{m}{2}\right]}\left(\begin{array}{c}
m \\
2 i
\end{array}\right) \partial_{0}^{m-2 i}\left(-\partial_{\underline{x}}\right)^{2 i}+\sum_{i=0}^{\left[\frac{m-1}{2}\right]}\left(\begin{array}{c}
m \\
2 i+1
\end{array}\right) \partial_{0}^{m-2 i-1}\left(-\partial_{\underline{x}}\right)^{2 i+1}
\end{aligned}
$$

the latter being splitted into a sum of scalar operators and a sum of vectorial operators, since $-\partial_{\underline{x}}^{2}=\Delta_{\underline{x}}$, where $\Delta_{\underline{x}}=\frac{\partial^{2}}{\partial x_{1}^{2}}+\ldots+\frac{\partial^{2}}{\partial x_{n}^{2}}$ is the Laplace operator in $\mathbb{R}^{n}$.

Then, for each $r \geq 0$, the equality (6) gives

$$
\begin{aligned}
\mathcal{P}_{r}{ }^{n}\left(\partial^{m}\right) \mathcal{P}_{k}{ }^{n}(x)= & \sum_{s=0}^{r}\left(\begin{array}{l}
r \\
s
\end{array}\right) c_{s}(n)\left(\sum_{i=0}^{\left[\frac{m}{2}\right]}\left(\begin{array}{c}
m \\
2 i
\end{array}\right) \partial_{0}^{m-2 i}\left(-\partial_{\underline{x}}\right)^{2 i}\right)^{r-s} \\
& \times\left(\sum_{i=0}^{\left[\frac{m-1}{2}\right]}\left(\begin{array}{c}
m \\
2 i+1
\end{array}\right) \partial_{0}^{m-2 i-1}\left(-\partial_{\underline{x}}\right)^{2 i+1}\right)^{s} \mathcal{P}_{k}^{n}(x) .
\end{aligned}
$$

Since $P_{k}(k=0,1,2, \ldots)$ are monogenic, then $-\partial_{\underline{x}} \mathcal{P}_{k}^{n}(x)=\partial_{0} \mathcal{P}_{k}^{n}(x)$ and we can replace $-\partial_{\underline{x}}$ by $\partial_{0}$ in the right-hand side of the above equality. For each $r \geq 0$, this procedure leads to

$$
\begin{aligned}
\mathcal{P}_{r}^{n}\left(\partial^{m}\right) \mathcal{P}_{k}^{n}(x)= & \sum_{s=0}^{r}\left(\begin{array}{l}
r \\
s
\end{array}\right) c_{s}(n)\left(\sum_{i=0}^{\left[\frac{m}{2}\right]}\left(\begin{array}{l}
m \\
2 i
\end{array}\right)\right)^{r-s} \\
& \times\left(\sum_{i=0}^{\left[\frac{m-1}{2}\right]}\left(\begin{array}{c}
m \\
2 i+1
\end{array}\right)\right) \partial_{0}^{m r} \mathcal{P}_{k}^{n}(x) \\
= & \sum_{s=0}^{r}\left(\begin{array}{l}
r \\
s
\end{array}\right) c_{s}(n) 2^{r(m-1)} \partial_{0}^{m r} \mathcal{P}_{k}^{n}(x),
\end{aligned}
$$

where the latter equality is coming from the known properties of the Pascal's triangle,

$$
\sum_{i=0}^{\left[\frac{m}{2}\right]}\left(\begin{array}{l}
m \\
2 i
\end{array}\right)=\sum_{i=0}^{\left[\frac{m-1}{2}\right]}\left(\begin{array}{c}
m \\
2 i+1
\end{array}\right)=2^{m-1}
$$

Observing that the monogenic sequence $\left(\mathcal{P}_{k}^{n}\right)_{k \geq 0}$ is Appell, it follows that

$$
\partial_{0}^{m r} \mathcal{P}_{k}^{n}(x)=\frac{k !}{(k-m r) !} \mathcal{P}_{k-m r}^{n}(x), \quad r \leq[k / m]
$$


and therefore, for each $0 \leq r \leq[k / m]$, we obtain

$$
\mathcal{P}_{r}{ }^{n}\left(\partial^{m}\right) \mathcal{P}_{k}{ }^{n}(x)=\sum_{s=0}^{r}\left(\begin{array}{l}
r \\
s
\end{array}\right) c_{s}(n) 2^{r(m-1)} \frac{k !}{(k-m r) !} \mathcal{P}_{k-m r}^{n}(x) .
$$

Then, for each $k \geq 0$, substituting this expression in (9), we obtain the monogenic generalized Hermite polynomials given by

$$
H_{k, m}^{(\lambda)}(x)=\sum_{r=0}^{[k / m]} \frac{1}{r !} \frac{k !}{(k-m r) !} \frac{\lambda^{r}}{2^{r}} \gamma_{r}(n) \mathcal{P}_{k-m r}^{n}(x),
$$

where $\gamma_{r}(n)=\sum_{s=0}^{r}\left(\begin{array}{l}r \\ s\end{array}\right) c_{s}(n)$ is the binomial transform of the sequence $\left(c_{s}\right)_{s \geq 0}$ with inverse $c_{r}(n)=\sum_{s=0}^{r}\left(\begin{array}{l}r \\ s\end{array}\right)(-1)^{r-s} \gamma_{s}(n)$ (see, e.g. [16]).

The constant polynomial $H_{0, m}^{(\lambda)}(x) \equiv 1$ (for any $m, \lambda$ ) is included in a natural way in (10), since $\gamma_{0}(n)=1$ and $\mathcal{P}_{0}^{n}(x) \equiv 1$, independently of the dimension $n$.

Special cases:

1. Real case $(n=0)$

Recalling that $c_{s}(0)=1(s=0,1,2, \ldots)$, it follows $\gamma_{r}(0)=\sum_{s=0}^{r}\left(\begin{array}{l}r \\ s\end{array}\right)=2^{r}$.

Taking into account also that $\mathcal{P}_{k}^{0}(x)=x_{0}^{k}$, from (10) we obtain the known generalized Hermite polynomials or Gould-Hopper polynomials in the real variable $x_{0}$,

$$
H_{k, m}^{(\lambda)}(x)=\sum_{r=0}^{[k / m]} \frac{1}{r !} \frac{k !}{(k-m r) !} \lambda^{r} x_{0}^{k-m r}
$$

2. Complex case $(n=1)$

For the case $n=1$, the polynomials $\mathcal{P}_{k}^{1}$ are isomorphic to the complex powers $z^{k}(k=0,1,2, \ldots)$ and $c_{s}(1)=1$, for arbitrary $s$. Therefore $\gamma_{r}(1)=2^{r}$ and $(10)$ is written now as

$$
H_{k, m}^{(\lambda)}(x) \cong \sum_{r=0}^{[k / m]} \frac{1}{r !} \frac{k !}{(k-m r) !} \lambda^{r} z^{k-m r}
$$

3. The special choices of $m=2$ and $\lambda=-\frac{1}{2}$ in (10) lead to

$$
H_{k}^{n}(x):=H_{k, 2}^{(-1 / 2)}(x)=\sum_{r=0}^{[k / 2]}(-1)^{r} \frac{1}{r !} \frac{k !}{(k-2 r) !} \frac{1}{4^{r}} \gamma_{r}(n) \mathcal{P}_{k-2 r}^{n}(x)
$$


which corresponds to the generalization for the hypercomplex case of the wellknown Hermite polynomials defined on the real line. In fact, if $\underline{x} \equiv 0$ (or $n=0$ ) then $\mathcal{P}_{k-2 r}^{0}(x)=x_{0}^{k-2 r}, \gamma_{r}(0)=1, \forall r$ and (11) has the form

$$
H_{k}^{0}(x)=H_{k, 2}^{(-1 / 2)}(x)=\sum_{r=0}^{[k / 2]}(-1)^{r} \frac{1}{r !} \frac{1}{2^{r}} \frac{k !}{(k-2 r) !} x_{0}^{k-2 r}
$$

4. The choices $m=2$ and $\lambda=-1$ in (10) as well as the consideration of the variable $2 x$ instead of $x$ give the polynomials

$$
H_{k, 2}^{(-1)}(2 x)=\sum_{r=0}^{[k / 2]}(-1)^{r} \frac{1}{r !} \frac{k !}{(k-2 r) !} \frac{1}{2^{r}} \gamma_{r}(n) \mathcal{P}_{k-2 r}^{n}(2 x)
$$

that for the particular case of $n=0$ (real case) coincides with the ordinary Hermite polynomials used frequently in physics and related to the Gaussian function $e^{-x_{0}^{2}}$.

The first hypercomplex Hermite polynomials (11) are given by

$$
\begin{aligned}
H_{0}^{n}(x) & =1 \\
H_{1}^{n}(x) & =\mathcal{P}_{1}^{n}(x) \\
& =x_{0}+\frac{1}{n} \underline{x} \\
H_{2}^{n}(x) & =\mathcal{P}_{2}^{n}(x)-\frac{1}{2}\left(1+\frac{1}{n}\right) \\
& =x_{0}^{2}+\frac{2}{n} x_{0} \underline{x}+\frac{1}{n} \underline{x}^{2}-\frac{1}{2}\left(1+\frac{1}{n}\right) \\
H_{3}^{n}(x) & =\mathcal{P}_{3}^{n}(x)-\frac{3}{2}\left(1+\frac{1}{n}\right) \mathcal{P}_{1}^{n} \\
& =x_{0}^{3}+\frac{3}{n} x_{0}^{2} \underline{x}+\frac{3}{n} x_{0} \underline{x}^{2}+\frac{3}{n(n+2)} \underline{x}^{3}-\frac{3}{2}\left(1+\frac{1}{n}\right)\left(x_{0}+\frac{1}{n} \underline{x}\right) .
\end{aligned}
$$

It is easy to show that analogously to the classical case also the hypercomplex Hermite polynomials form an Appell sequence, i.e.,

$$
\frac{1}{2} \partial H_{k}^{n}(x)=k H_{k-1}^{n}(x), \quad k \geq 1
$$

and, therefore, they satisfy the binomial-type theorem

$$
H_{k}^{n}(x)=H_{k}^{n}\left(x_{0}+\underline{x}\right)=\sum_{r=0}^{k}\left(\begin{array}{l}
k \\
r
\end{array}\right) x_{0}^{r} H_{k-r}^{n}(\underline{x}) .
$$




\section{Monogenic Chebyshev polynomials of first and second kinds}

On the real line, the well-known Chebyshev polynomials of first kind $T_{k}$ and second kind $U_{k}$ can be explicitly defined by

$$
T_{k}(x)=\frac{k}{2} \sum_{r=0}^{[k / 2]}(-1)^{r} \frac{(k-1-r) !}{r !(k-1-2 r) !}(2 x)^{k-2 r}
$$

and

$$
U_{k}(x)=\sum_{r=0}^{[k / 2]}(-1)^{r} \frac{(k-r) !}{r !(k-2 r) !}(2 x)^{k-2 r}
$$

An interesting link between these polynomials and the Gould-Hopper polynomials was made in [7] using the integral representation of $k$ ! provided by the Gamma function i.e., $k !=\int_{0}^{\infty} e^{-t} t^{k} d t$. We can follow an analogous procedure to construct the hypercomplex analogues of the Chebyshev polynomials by considering the monogenic generalized Hermite polynomials (10) with the choice of the parameters $m=2$ and $\lambda=-\frac{1}{t}(t>0)$ and considering the variable $2 x$ instead of $x$,

$$
H_{k, 2}^{(-1 / t)}(2 x)=\sum_{r=0}^{[k / 2]}(-1)^{r} \frac{k !}{r !(k-2 r) !} \frac{1}{t^{r} 2^{r}} \gamma_{r}(n) \mathcal{P}_{k-2 r}^{n}(2 x)
$$

Multiplying each summand by $e^{-t} t^{k}\left(t \in \mathbb{R}^{+}\right)$and integrating, we get

$$
\begin{aligned}
\int_{0}^{\infty} e^{-t} t^{k} H_{k, 2}^{(-1 / t)}(2 x) d t & =\sum_{r=0}^{[k / 2]}(-1)^{r} \frac{k !}{r !(k-2 r) !} \int_{0}^{\infty} e^{-t} t^{k-r} d t \frac{1}{2^{r}} \gamma_{r}(n) \mathcal{P}_{k-2 r}^{n}(2 x) \\
& =\sum_{r=0}^{[k / 2]}(-1)^{r} \frac{k !(k-r) !}{r !(k-2 r) !} \frac{1}{2^{r}} \gamma_{r}(n) \mathcal{P}_{k-2 r}^{n}(2 x) .
\end{aligned}
$$

The monogenic Chebyshev polynomials of second kind can be defined as

$$
\begin{aligned}
U_{k}^{n}(x) & :=\frac{1}{k !} \int_{0}^{\infty} e^{-t} t^{k} H_{k, 2}^{(-1 / t)}(2 x) d t \\
& =\sum_{r=0}^{[k / 2]}(-1)^{r} \frac{(k-r) !}{r !(k-2 r) !} \frac{1}{2^{r}} \gamma_{r}(n) \mathcal{P}_{k-2 r}^{n}(2 x),
\end{aligned}
$$

For the cases $n=0$ and $n=1$ we obtain the Chebyshev polynomials of second kind in the real and complex variables, respectively, as particular cases of $U_{k}^{n}$ $(k=0,1,2, \ldots)$.

The monogenic Chebyshev polynomials of first kind can be obtained from the generalized Hermite polynomials (10) for the same choices of the parameters $m$ 
and $\lambda$ and the same scaled variable $2 x$. Now, we consider the equality $(k-1) !=$ $\int_{0}^{\infty} e^{-t} t^{k-1} d t$ and in analogous way, we get

$$
\int_{0}^{\infty} e^{-t} t^{k-1} H_{k, 2}^{(-1 / t)}(2 x) d t=k ! \sum_{r=0}^{[k / 2]}(-1)^{r} \frac{(k-1-r) !}{r !(k-2 r) !} \frac{1}{2^{r}} \gamma_{r}(n) \mathcal{P}_{k-2 r}^{n}(2 x)
$$

Defining the monogenic Chebyshev polynomials of first kind by

$$
\begin{aligned}
T_{k}^{n}(x) & :=\frac{1}{2(k-1) !} \int_{0}^{\infty} e^{-t} t^{k-1} H_{k, 2}^{(-1 / t)}(2 x) d t \\
& =\frac{k}{2} \sum_{r=0}^{[k / 2]}(-1)^{r} \frac{(k-1-r) !}{r !(k-2 r) !} \frac{1}{2^{r}} \gamma_{r}(n) \mathcal{P}_{k-2 r}^{n}(2 x), \quad k=1,2, \ldots
\end{aligned}
$$

we obtain the Chebyshev polynomials of first kind in the real and complex variables as particular cases of $T_{k}^{n}(k=0,1,2, \ldots)$ for $n=0$ and $n=1$, respectively.

In consequence of the homogeneity of the polynomials $\mathcal{P}_{k}^{n}$ and the fact that they form an Appell sequence, the monogenic Chebyshev polynomials of first and second kinds are related by the equality

$$
\frac{1}{2} \partial T_{k}(x)=k U_{k-1}(x), \quad k=1,2, \ldots
$$

\section{Concluding remarks}

The construction of Hermite polynomials in the framework of Clifford algebras was considered earlier by some authors. The so-called radial Hermite polynomials were first constructed in [18] using the Cauchy-Kowalevskaya extension of a suitable chosen generalization of the generating function of the Hermite polynomials on the real line. The obtained polynomials are functions of a purely vectorial argument as the next first examples show,

$$
\begin{aligned}
& H_{0}(\underline{x})=1 \\
& H_{1}(\underline{x})=\underline{x} \\
& H_{2}(\underline{x})=\underline{x}^{2}+n \\
& H_{3}(\underline{x})=\underline{x}^{3}+(n+2) \underline{x} .
\end{aligned}
$$

Also in [3] and using a different approach, another generalization of the Hermite polynomials was considered in Clifford Algebras over the complex field. The obtained polynomials are also functions of a pure vector argument and therefore as before a direct compatibility with the real or complex case is not visible. Our main motivation was to obtain monogenic generalizations of Hermite polynomials that contain the real and the holomorphic cases as particular cases. Moreover, the construction of generalized Hermite polynomials with varying orders and parameters permitted to construct the monogenic Chebyshev polynomials in an easy way. 


\section{Acknowledgments}

Financial support from "Center for research and development in Mathematics and Applications" of the University of Aveiro, through the Portuguese Foundation for Science and Technology (FCT), is gratefully acknowledged.

\section{References}

1. Bock, S., Gürlebeck, K.: On a generalized Appell system and monogenic power series. Math. Methods Appl. Sci. 33(4), 394-411 (2010)

2. Brackx, F.: The exponential function of a quaternion variable. Applicable Anal. 8(3), 265-276 (1978/79)

3. Brackx, F., De Schepper, N., Sommen, F.: Clifford algebra-valued orthogonal polynomials in Euclidean space. J. Approx. Theory 137(1), 108-122 (2005), http://dx.doi.org/10.1016/j.jat.2005.08.004

4. Brackx, F., Delanghe, R., Sommen, F.: Clifford analysis. Pitman, Boston-LondonMelbourne (1982)

5. Cação, I., Falcão, M.I., Malonek, H.R.: Laguerre derivative and monogenic Lag uerre polynomials: an operational approach. Mathematical and Computer Modelling (2010)

6. Cação, I., Malonek, H.: On complete sets of hypercomplex Appell polynomials. In: Simos, T.E., Psihoyios, G., Tsitouras, C. (eds.) AIP Conference Proceedings. vol. 1048, pp. 647-650 (2008)

7. Dattoli, G.: Laguerre and generalized Hermite polynomials: the point of view of the operational method. Integral Transforms Spec. Funct. 15(2), 93-99 (2004), http://dx.doi.org/10.1080/10652460310001600744

8. Falcão, M.I., Cruz, J., Malonek, H.R.: Remarks on the generation of monogenic functions. 17th Inter. Conf. on the Appl. of Computer Science and Mathematics on Architecture and Civil Engineering, Weimar (2006)

9. Falcão, M.I., Malonek, H.R.: Generalized exponentials through Appell sets in $\mathbb{R}^{n+1}$ and Bessel functions. In: Simos, T.E., Psihoyios, G., Tsitouras, C. (eds.) AIP Conference Proceedings. vol. 936, pp. 738-741 (2007)

10. Gould, H.W., Hopper, A.: Operational formulas connected with two generalizations of hermite polynomials. Duke Math. J. 29, 51-62 (1962)

11. Gürlebeck, K., Malonek, H.: A hypercomplex derivative of monogenic functions in $\mathbb{R}^{n+1}$ and its applications. Complex Variables Theory Appl. 39, 199-228 (1999)

12. Lávička, R.: Canonical bases for $\mathrm{sl}(2, \mathrm{c})$-modules of spherical monogenics in dimension 3 (2010)

13. Malonek, H.: A new hypercomplex structure of the euclidean space $\mathbb{R}^{m+1}$ and the concept of hypercomplex differentiability. Complex Variables 14, 25-33 (1990)

14. Malonek, H.: Selected topics in hypercomplex function theory. In: Eriksson, S.L. (ed.) Clifford algebras and potential theory, pp. 111-150. 7, University of Joensuu (2004)

15. Malonek, H.R., Falcão, M.I.: Special monogenic polynomials-properties and applications. In: Simos, T.E., Psihoyios, G., Tsitouras, C. (eds.) AIP Conference Proceedings. vol. 936, pp. 764-767 (2007)

16. Riordan, J.: Combinatorial identities. John Wiley \& Sons Inc., New York (1968)

17. Sommen, F.: A product and an exponential function in hypercomplex function theory. Appl. Anal. 12, 13-26 (1981) 
18. Sommen, F.: Special functions in clifford analysis and axial symmetry. J. Math. Anal. Appl. 130(1), 110-133 (1988) 